\title{
Alleviation of Exercise-induced Dehydration under Hot Conditions by Glycerol Hyperhydration
}

\author{
Takeshi Nishijima*, Hirokazu Tashiro ${ }^{* *}$, Morimasa Kato ${ }^{* * *}$, Tsuyoshi Saito ${ }^{* * *}$, Takenori Omori*, \\ Hyukki Chang $^{*}$, Nao Ohiwa ${ }^{* * * * *}$, Yousuke Sakairi ${ }^{* * * * *}$ and Hideaki Soya ${ }^{*}$ \\ *Exercise Biochemistry, University of Tsukuba Graduate School of Comprehensive Human Sciences \\ 1-1-1 Tennodai, Tsukuba, Ibaraki 305-8574 Japan \\ hsoya@taiiku.tsukuba.ac.jp \\ *** Master's Program in Health and Sport Sciences, University of Tsukuba \\ 1-1-1 Tennodai, Tsukuba, Ibaraki 305-8574 Japan \\ *** Department of Health and Nutrition, Yonezawa Women's Junior College of Yamagata Prefecture \\ 6-15-1, Torimachi, Yonezawa, Yamagata 992-0025 Japan \\ **** Department of Welfare Psychology Shizuoka University of Welfare \\ 549-1, Honnakane, Yaizu, Shizuoka 425-0063 Japan \\ ***** Department of Sports Sciences, Japan Institute of Sports Sciences \\ 3-15-1, Nishigaoka, Kita-ku, Tokyo 115-0056 Japan \\ ${ }^{* * * * * * *}$ Psychology of Sports and Physical Education, University of Tsukuba Graduate School of Comprehensive Human Sciences \\ 1-1-1 Tennodai, Tsukuba, Ibaraki 305-8574 Japan \\ [Received May 1, 2006 ; Accepted January 10, 2007]
}

\begin{abstract}
Ingestion of glycerol with a large volume of fluid causes hyperhydration, which has been shown to increase thermoregulatory capacity and improve exercise performance under hot conditions, but in some cases causes nausea and headaches. However, it is still uncertain what kind of hydration regimen would be effective in producing a greater hyperhydration state and whether glycerol hydration would affect mood. This study compared the hyperhydration effectiveness of different hydration regimens, then examined whether glycerol hyperhydration prior to exercise alleviates exercise-induced dehydration, improves exercise performance under hot conditions, and affects mood while hydrated. Both the Bolus- $G$ regimen: ingesting a relatively high concentration of glycerol fluid at the beginning of the hydration phase, and the $G$ regimen: ingesting a low concentration of glycerol fluid through hydration phase, caused significant increase in body weight and decrease total urine volume, but there was no significant differences between two regimens. Ninety minutes after glycerol or placebo hydration by the Bolus-G regimen, six healthy well-trained volunteers performed a 70 -min cycling test. The decrease of body weight after exercise versus pre-hydration was significantly smaller in the glycerol trial $(1.4 \%$ of body weight) than in the placebo trial $(2.5 \%)$. There were no significant differences in exercise performance and mood measured by a two-dimensional mood scale during the hydration phase. These results suggest that hyperhydration by glycerol alleviates exercise-induced dehydration during exercise in a hot environment but with no changes in exercise performance and mood.
\end{abstract}

Keywords: hydration, sweat, exercise performance, mood, two-dimensional mood scale

[International Journal of Sport and Health Science Vol.5, 32-41, 2007]

\section{Introduction}

Exercise performance will deteriorate because of dehydration caused by heavy sweating during exercise under hot conditions (Walsh, et al., 1994). In addition, excessive dehydration could increase the risk of heat stroke during sports activities. Although proper hydration is fundamental in order to prevent dehydration during exercise (Sawka, 1992; McConell, et al., 1997), it is difficult for an athlete to make up for all their lost fluids during a period of intense sweating under hot conditions even if they actively hydrate (Yorimoto, et al., 1995). It is not easy to prevent dehydration during exercise by hydration only because hydration opportunities are often limited in certain sports (Burke and Hawley, 1997). Pre-exercise hyperhydration through glycerol loading (glycerol and fluid ingestion) has been proposed as one effective measure to cope with this problem. The definition of hyperhydration is for the 
body to be in a state with more water content than usual. It is reported that pre-exercise hyperhydration can enhance thermoregulatory functions during exercise under hot conditions (Lyons, et al., 1990) and improve exercise performance (Montner, et al., 1996; Hitchins, et al., 1999; Anderson, et al., 2001). Preventing dehydration during exercise is important in Japan which is known for hot and humid conditions. However, glycerol loading is less recognized in Japan and few studies have been conducted on it so far.

Glycerol is a lipid with three carbon atoms. It is an osmotically active substance existing in all cells and in low concentrations in extracellular fluid in vivo $(<0.1 \mathrm{mmol} / 1)$ (Robergs and Griffin, 1998). In general in glycerol loading 1-1.5g per kilogram of body weight of glycerol is taken with plenty of fluid (20-26ml per kilogram of body weight) (Robergs and Griffin, 1998). As a result body water content increases by 300-700 $\mathrm{ml}$ (Robergs and Griffin, 1998) and a duration of more than four hours of hyperhydration can be achieved (Riedesel, et al., 1987). In contrast, when taking plenty of water or sports drinks without glycerol, diuresis is induced and hyperhydration does not occur.

Although several studies have so far reported improvements in performance during exercise under hot conditions through pre-exercise glycerol loading (Montner, et al., 1996; Hitchins, et al., 1999; Anderson, et al., 2001), there also have been reports of its ineffectiveness (Marino, et al., 2003). Differences in hydration methods and the amount of pre-exercise water retention in each study may have caused these different results. For instance, in the study of Marino, et al., (2003), which reported no improvement of exercise performance, a water retention effect was not sufficient because the water retention amount was as little as $300 \mathrm{ml}$. However, water retention effects caused by differences in glycerol loading methods have never been compared in the same experiment in any study, and there is only one documented comparison (Robergs and Griffin, 1998). When examining whether glycerol loading can alleviate dehydration and improve exercise performance during exercise under hot conditions, it is first necessary to identify a glycerol loading method which can increase the water retention amount. In addition, it is also reported that excessive glycerol fluid uptake causes headaches and blurred vision (Murrai, et al., 1991; Burke, 2001). These symptoms might negatively affect mood, although there has not been any report on the effect on mood as yet.

The present study first compares the effectiveness of two typical glycerol loading methods (after Robergs and Griffin, 1998) (Experiment 1). Then, the glycerol loading method whose effectiveness is observed in Experiment 1 is checked to see if it can alleviate dehydration during longtime exercise under hot conditions (Experiment 2). In Experiment 2, the effect of glycerol loading on mood, exercise performance and physiological parameters such as heart rate is also examined. Changes in mood are evaluated by using a two-dimensional mood scale (Sakairi, et al., 2003a; 2003b) which allows continuous monitoring. A seventy minute cycling test was used with exercise performance being evaluated during the last 30-minute period.

\section{Methodology}

All experiments were conducted based on the regulations of the Research Ethics Committee of the Institute of Health and Sport Sciences, University of Tsukuba. The subjects were fully explained of the purpose and contents of this experiment, and the experiments were started after obtaining prior consent from them.

\subsection{Glycerol loading methods (Experiment 1)}

\subsubsection{Subjects}

The subjects of this study were healthy male students (age: $22.8 \pm 1.4$, height: $171.6 \pm 4.5 \mathrm{~cm}$, weight: $63.98 \pm 6.14 \mathrm{~kg}$, mean value \pm standard deviation).

\subsubsection{Experiment protocol}

Each subject was hydrated through three different glycerol loading methods chosen at random, each with at least a two day interval. In order to participate in the experiment with sufficient initial body water retention, subjects were instructed to take more than $8 \mathrm{ml}$ of water per kilogram in body weight one hour before starting the experiment. After arriving at the laboratory, subjects were told to urinate and defecate and then their nude body weights were measured (20g accuracy, A\&D, FW-150K). After resting for ten minutes, the subjects started hydration through one of the three protocols shown 
Table 1 Hydration protocols

\begin{tabular}{|c|c|}
\hline \multicolumn{2}{|l|}{ HW protocol } \\
\hline $0-60 \min$ & HW $25 \mathrm{ml} / \mathrm{kg}$ \\
\hline \multicolumn{2}{|l|}{ G protocol } \\
\hline $0-60 \mathrm{~min}$ & Glycerol $1.2 \mathrm{~g} / \mathrm{kg}+\mathrm{HW} 25 \mathrm{ml} / \mathrm{kg}$ \\
\hline \multicolumn{2}{|c|}{ Bolus-G protocol } \\
\hline $0-30 \mathrm{~min}$ & Glycerol $1.0 \mathrm{~g} / \mathrm{kg}+\mathrm{HW} 8 \mathrm{ml} / \mathrm{kg}$ \\
\hline $30-45$ & $\mathrm{HW} 4 \mathrm{ml} / \mathrm{kg}$ \\
\hline $45-60$ & $\mathrm{HW} 4 \mathrm{ml} / \mathrm{kg}$ \\
\hline $60-75$ & Glycerol $0.2 \mathrm{~g} / \mathrm{kg}+\mathrm{HW} 5 \mathrm{ml} / \mathrm{kg}$ \\
\hline $75-90$ & HW 4 ml/kg \\
\hline
\end{tabular}

below. Ninety minutes after completing hydration, subjects were told to urinate and their nude body weights were again measured. Urination during the experiment was at the discretion of each subject. All urine volumes were recorded from the beginning of hydration until 90 minutes after completing it, in order to determine the total urine volume. All experiments were conducted from 9 am to $1 \mathrm{pm}$.

Hypotonic water (HW) was used in all drinks in the experiment, which was diluted twice from a sports drink (Aquarius, Coca-Cola Co., Ltd., carbohydrate: $4.7 \mathrm{~g}$; protein: $0 \mathrm{~g}$; fat: $0 \mathrm{~g}$; sodium: $34 \mathrm{mg}$; magnesium: $1.2 \mathrm{mg}$; arginine: $25 \mathrm{mg}$; isoleucine: $1 \mathrm{mg}$; valine: $0.5 \mathrm{mg}$ per $100 \mathrm{ml}$ ). The following three protocols were established as hydration methods (Table 1). 1) HW is taken in 60 min (HW); 2) HW containing $1.2 \mathrm{~g}$ of glycerol (a designated food additive supplied by Kishida Chemical Co., Ltd.,) per kilogram of body weight is taken in 60 min $(G), 3)$ High concentration glycerol ( $1.0 \mathrm{~g}$ per kilogram) is taken at first and then $\mathrm{HW}$ is taken in 90 min (Bolus-G). The Bolus-G protocol established by Montner, et al., (1996) is considered tobe the most effective water intake method for internal water retention (Robergs and Griffin, 1998). In contrast, $G$ protocol, where a specific concentration of glycerol solution is taken, has been used in many other studies (Hitchins, et al., 1999; Anderson, et al., 2001; Marino, et al., 2003. The total water amount taken in each protocol was determined to be $25 \mathrm{ml}$ per kilogram of body weight, and the total glycerol amount taken in $\mathrm{G}$ and Bolus-G protocols to be $1.2 \mathrm{~g}$ per kilogram of body weight. In HW and
$\mathrm{G}$ protocols, subjects were instructed to take at a constant pace within the time frame.

\subsection{Effect of glycerol loading on dehydration, exercise performance and mood (Experiment 2)}

\subsubsection{Subjects}

The subjects were seven university middle-distance runners. One of the subjects became ill and could not finish the experiment so the results of six subjects (age: $19.3 \pm 0.2$ years, height: $173.7 \pm 2.3 \mathrm{~cm}$, weight: $66.04 \pm 1.97 \mathrm{~kg}$, mean $\pm \mathrm{SD}$ ) were analyzed.

\subsubsection{Setting of work load}

In order to determine the relative intensity of work load used in the exercise test, $\mathrm{VO}_{2}$ max and LT (lactate threshold) were measured two weeks before the exercise test. A cycling test was performed using a cycle ergometer (Strength-ergo 240, Mitsubishi Electric Corporation) and respiratory gas and blood lactate levels were measured during incremental-load exercise until the subjects felt completely exhausted. Subjects were told to warm-up freely after arriving at the laboratory and then indwelling needles ( 22 gage, Terumo Corporation) were inserted into their median antebrachial. After resting for 10 minutes, they were asked to start the incremental-load cycling exercise. The initial work load was $0 \mathrm{~N} \cdot \mathrm{m}$, which was increased by $4 \mathrm{~N} \cdot \mathrm{m}$ every three minutes during the first 18 minutes and every one minute after that. At the point when maintaining a rotation frequency of $60 \mathrm{rpm}$ became impossible, which is regarded as complete exhaustion, subjects were told to end the cycling exercise. Oxygen uptake during exercise was measured from respiratory gas taken through a face mask worn by each subject by using an automatic respiratory gas analyzer (AE-300S, Minato Medical Science Co., Ltd.). Blood samples were taken immediately before increasing work load and blood lactate levels were quickly measured by using a glucose/lactate automatic analyzer (YSI, Model 2300) to estimate LT (Beaver, et al., 1985). Heart rates were measured using a HR monitor (PORAR) and ratings of perceived exertion (RPE) (Borg, 1970) were recorded immediately before increasing work load.

Subjects were required to perform cycling exercise ( $60 \mathrm{rpm}$ rotation frequency) at $80 \%$ intensity of LT one week before the exercise test and the work load used in the exercise test was determined based on the 
following criteria. 1) Rotation frequency of $60 \mathrm{rpm}$ can be maintained for $40 \mathrm{~min}$. 2) Cycling exercise can be continued for 60 minutes without any extreme slowing down after cycling for 40 minutes. When these criteria could not be achieved, the work load was decreased so that cycling could continue.

\subsubsection{Exercise test protocol}

A double-blind and cross-over design was used in the tests, which were performed in a laboratory with adjusted room temperature $\left(30.3 \pm 0.6{ }^{\circ} \mathrm{C}\right.$, mean \pm SD) and humidity $(50.8 \pm 2.3 \%$, mean \pm SD $)$ with an interval of a week. The Bolus-G protocol was used for the hydration method. Subjects took glycerol drinks or placebo drinks within $90 \mathrm{~min}$ and cycling exercise was started 90 min after completing hydration. Since glycerol is sweet, the taste of placebo drink was adjusted by using calorie-free sweetener (Erythritol, Asadaame Co., Ltd.). Subjects were told to take Calorie Mate (400 kcal, Otsuka Pharmaceutical Co., Ltd), Weider in Jelly (180 kcal, Morinaga \& Co., Ltd) and more than $8 \mathrm{ml}$ fluid per kilogram of body weight up to one hour before the test. Moreover, any hard training leading to complete exhaustion was prohibited within the two days prior to the test. Training on the experiment day, smoking and taking caffeine and alcohol on the previous day were also prohibited.

In order to enable sequential blood sampling, an indwelling needle was inserted into the median artebrachial of each subject after arriving at the laboratory. After a 10-min rest in a sitting posture, subjects were told to urinate, measure nude body weight and start hydration. While subjects were allowed to urinate at their own will during hydration, all subjects were told to urinate immediately before starting exercise. Urine volume was recorded for each urination from the beginning of hydration to just before the beginning of exercise to determine the total urine volume. Plasma osmolality was measured three times from sampled blood by using an automatic osmotic pressure meter (ONE-TEN Osmo-meter, FISK): before hydration, 90 min later; and, $180 \mathrm{~min}$ later. Subjects were allowed to perform warm-up exercise freely $180 \mathrm{~min}$ after starting hydration (90 min after hydration ended) and then told to start the cycling exercise.

The cycling exercise consists of a three-minutes warm-up ( $50 \%$ of specified load intensity), a 40 min phase pedaling with a rotation frequency of
$60 \mathrm{rpm}$ (fixed power phase) and a 30-min phase pedaling at maximum power (variable power phase) (Jeukendrup, et al., 1996; Hitchins, et al., 1999). The exercise performance of each subject was evaluated from the total work load $(\mathrm{kJ})$ at the variable power phase. Blood sampling was conducted every 10 min after exercise started. Blood glucose and blood lactate levels were quickly measured using a glucose/lactate automatic analyzer. Plasma osmolality was measured $40 \mathrm{~min}$ after starting exercise and after completing exercise. RPE and graded classifications of temperature sensibility were measured every 10 min using a modified version of the Young, et al., (1987) scale (0: unbearably cold, 8: unbearably hot). Thirst was also measured every 10 mins using a modified version of the Murray et al. (1991) scale (0: no thirst, 8: very thirsty). Heart rates were recorded every $5 \mathrm{~min}$. The drum temperature was measured before and after exercise by using a drum thermometer (BT-09, Nishitomo Co., Ltd.). When the exercise was completed, the nude body weights of subjects were measured after urination, and the amount of dehydration was evaluated from body weight shrinkage compared to the weight at the beginning of hydration. Subjects were not informed of data such as heart rate nor encouraged to do their best, but were only told of the remaining time during exercise. In addition, no hydration was given at all during exercise.

Mood during hydration and during exercise was recorded using a two-dimensional mood scale developed by Sakairi et al. (2003a, 2003b). Measurements were taken before hydration and then every 30 min during the three hours of hydration until just before starting exercise, $40 \mathrm{~min}$ after starting exercise and at the end of exercise. The two-dimensional mood scale classifies four different types of mood; positive arousal, negative arousal, arousal and hedonic. It is based on eight question items with a range from -10 to +10 points. The psychological state of each subject on the experiment day was recorded using POMS (profile of mood states) before starting hydration.

\subsection{Statistical analysis}

All the data are shown by mean \pm SD. In Experiment 1, a one-way analysis of variance was conducted to do multiple comparison tests (Scheffé). In Experiment 2, a paired $t$-test was conducted to test 


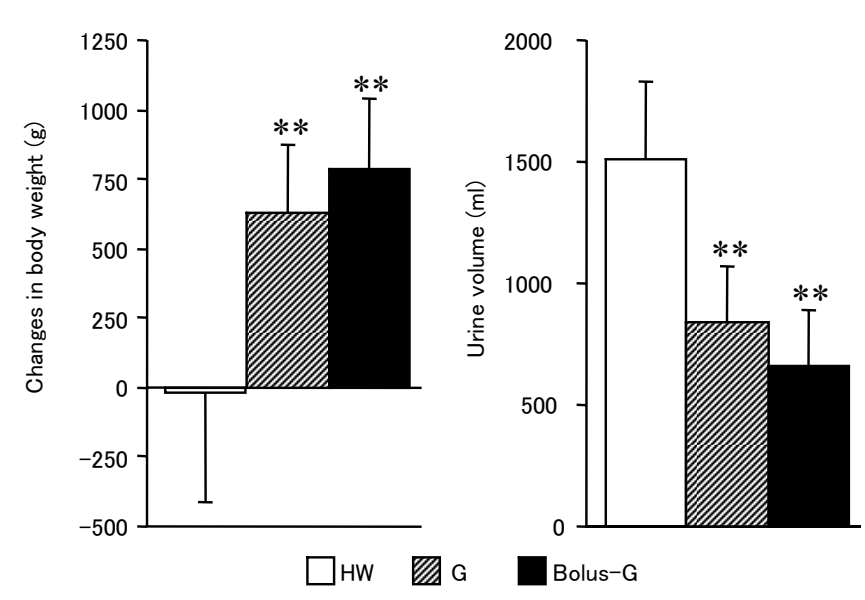

Figure 1 Effects of hydration on changes in body weight (left) and total urine volume (right). Data are expressed as mean $\pm \mathrm{SD}(\mathrm{n}=10) . * * p<0.01$

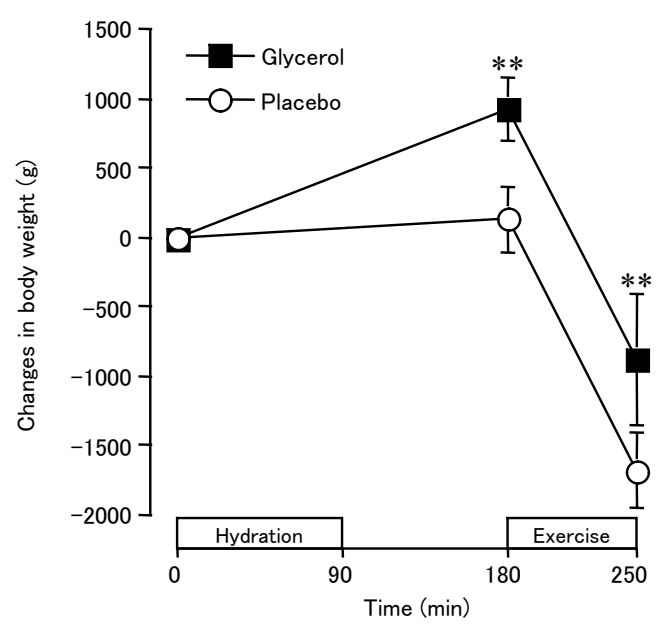

Figure 2 Changes in body weight in glycerol and placebo trials over time. Data are expressed as mean $\pm \mathrm{SD}(\mathrm{n}=6)$. $* * p<0.01$ vs. placebo trial.

the difference between two groups and a two-way analysis of variance to test the difference among multiple groups. The major effects caused by differences in the hydration methods are examined. The criterion of statistical significance was set below the 5\% level. All statistical analysis was performed using SPSS 12.0J for Windows (SPSS Incorporation).

\section{Results}

\subsection{Water retention effect due to difference in glycerol loading methods (Experiment 1)}

Figure 1 shows changes in body weight (left) and total urine volume (right) when conducting water intake in three different methods. Changes in
Table 2 Physiological characteristics and work load in exercise test

\begin{tabular}{ccccc}
\hline \multirow{2}{*}{ ID } & \multirow{2}{*}{$\begin{array}{c}\mathrm{VO}_{2} \mathrm{max} \\
(\mathrm{ml} / \mathrm{min} / \mathrm{kg})\end{array}$} & $\begin{array}{c}\mathrm{LT} \\
(\mathrm{N} \cdot \mathrm{m})\end{array}$ & \multicolumn{2}{c}{ Work Load } \\
\cline { 3 - 5 } & 60.6 & 36 & 27 & 75 \\
2 & 62.2 & 36 & 27 & 75 \\
3 & 65.5 & 34 & 23 & 68 \\
4 & 64.0 & 36 & 25 & 69 \\
5 & 60.6 & 40 & 29 & 73 \\
6 & 54.7 & 32 & 24 & 78 \\
\hline \hline
\end{tabular}

Mean \pm SD. $\quad 61.3 \pm 3.7 \quad 35.7 \pm 2,7 \quad 26.0 \pm 2.1 \quad 73.0 \pm 3.9$

body weight were HW: $-12 \pm 402 \mathrm{~g}$, G: $619 \pm 251 \mathrm{~g}$, Bolus-G: $774 \pm 268 \mathrm{~g}$. There were significantly higher values in $\mathrm{G}(p<0.01)$ and Bolus- $\mathrm{G}(p<0.01)$ compared to HW. Urine volumes were HW: 1497 $\pm 337 \mathrm{ml}$, G: $826 \pm 244 \mathrm{ml}$, Bolus-G: $654 \pm 235 \mathrm{ml}$, with significantly lower values in $\mathrm{G}(p<0.01)$ and Bolus-G $(p<0.01)$ compared to HW. No statistically significant difference was observed in changes in body weight and total urine volume between $\mathrm{G}$ and Bolus-G. Although three out of six subjects reported minor headaches and blurred vision during the glycerol drink intake, these subjective symptoms gradually became moderate after the end of hydration and almost disappeared $60 \mathrm{~min}$ after that.

\subsection{Effect of glycerol loading on dehydration, exercise performance and mood during exercise (Experiment 2)}

Table 2 shows the physiological characteristics of subjects in Experiment 2. The average work load used in the exercise test was $26.0 \pm 2.1 \mathrm{~N} \cdot \mathrm{m}(73.0 \pm$ $3.9 \%$ LT intensity).

Figure 2 shows changes in body weight over time from the start of hydration to the end of exercise. Similarly to Experiment 1, body weight increased due to glycerol fluid intake - the Glycerol group $(920 \pm$ $229 \mathrm{~g})$ showed significantly higher values $(p<0.01)$ compared to the Placebo group $(130 \pm 239 \mathrm{~g})$. After 70-min cycling exercise, body weight decreased considerably in both groups, and no difference in body weight shrinkage due to exercise was observed between the Glycerol group $(1807 \pm 274 \mathrm{~g})$ and the Placebo group $(1807 \pm 307 \mathrm{~g})$. The Glycerol group $(-887 \pm 473 \mathrm{~g})$ showed significantly higher values $(p<$ $0.01)$ compared to the Placebo group $(-1676 \pm 267 \mathrm{~g})$ in body weight at the end of exercise. The shrinkage 


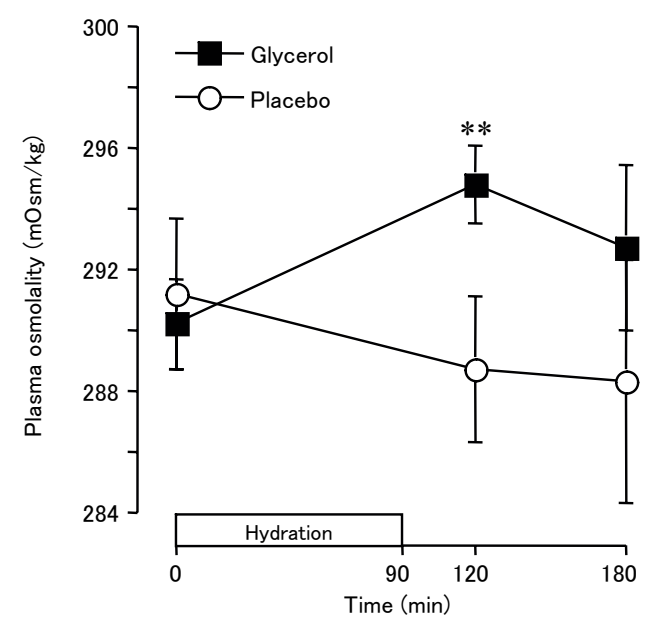

Figure 3 Changes in plasma osmolality from 0 min (start of hydration) to 180 (start of exercise) in glycerol and placebo trials. Data are expressed as mean $\pm \mathrm{SD}(\mathrm{n}=6)$.

$* * p<0.01$ vs. placebo trial.

of body weight compared to weight before water intake was $1.4 \pm 0.9 \%$ of body weight in the Glycerol group, while it was $2.5 \pm 0.4 \%$ of body weight in the Placebo group. The Glycerol group (560 \pm $212 \mathrm{ml})$ showed significantly lower values $(p<0.01)$ compared to Placebo group $(1224 \pm 257 \mathrm{ml})$ in the urine volume immediately before the start of exercise (data not shown).

Figure 3 shows change in plasma osmolality over time from the start of hydration to immediately before starting exercise $(180 \mathrm{~min})$. No difference between the groups was observed in plasma osmolality before the start of hydration. While plasma osmolality increased with glycerol fluid intake, it decreased with placebo fluid intake. The Glycerol group $(294.8 \pm 1.3 \mathrm{mOsm} / \mathrm{kg})$ showed significantly higher values $(p<0.01)$ compared to the Placebo group $(288.7 \pm 2.4 \mathrm{mOsm} / \mathrm{kg})$ after $120 \mathrm{~min}$ passed.

Figure 4 shows changes in average power output (top) every 5 min during the 70-min of cycling exercise and the total work load at the variable power phase (below) during the latter $30 \mathrm{~min}$. The average power output at the fixed power phase during the earlier 40 min was constant for both groups. The Glycerol group showed higher values regarding average power output at the variable power phase but no significant difference was observed. The total work load at the variable power phase increased by $9 \%$ on average in the Glycerol group. Although there were increases in two subjects out of the six, three subjects did not change and one decreased.
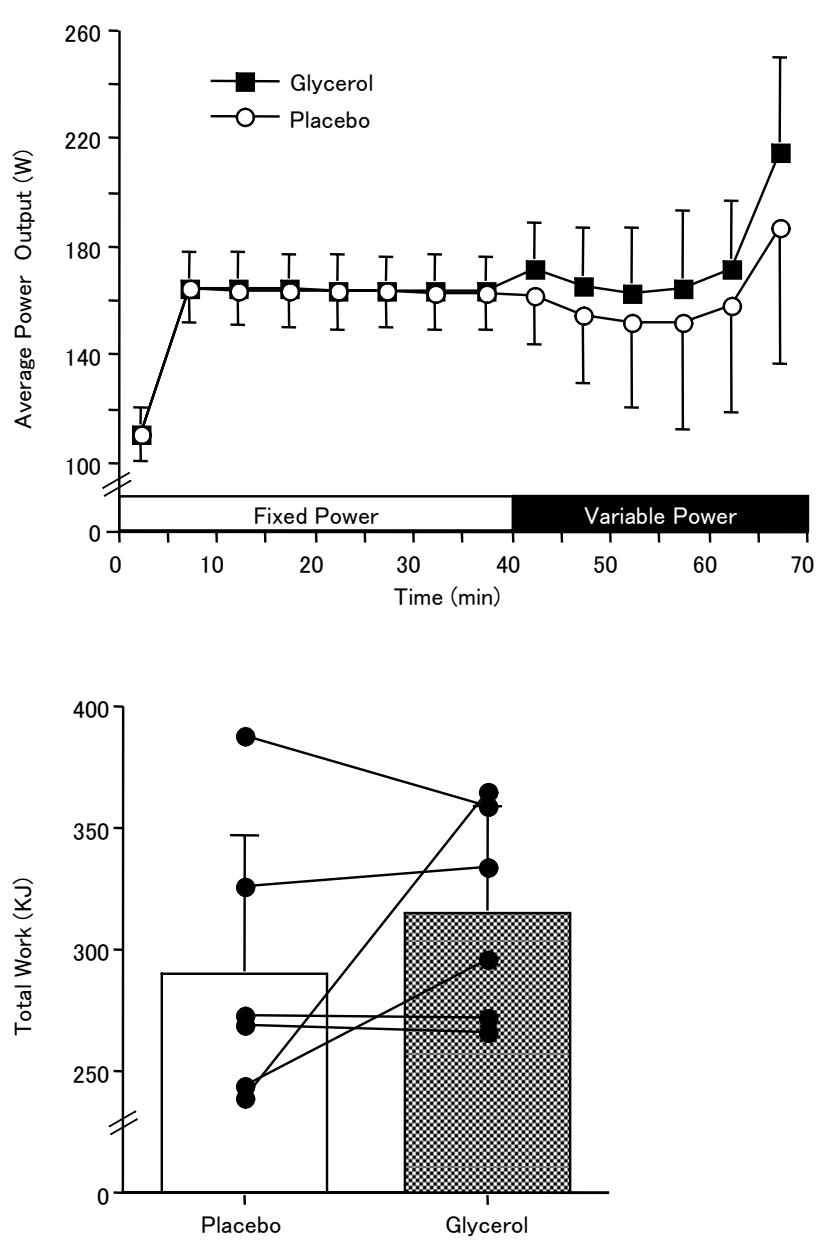

Figure 4 Changes in averaged power output (top) and total work in the variable power phase (bottom) in glycerol and placebo performance trials. Data are expressed as mean \pm SD $(n=6)$.

As a result, no significant difference was observed between the groups.

Figure 5 shows changes in heart rate (HR), glucose level, lactate level and plasma osmolality over time during cycling exercise. No significant difference was observed in any physiological parameter between the groups. Similarly, there was no significant difference in RPE, temperature sensibility, thirst and drum temperature between the groups (data not shown).

Figure 6 shows changes in mood from the start of hydration to the end of cycling exercise. This is classified into four attributes measured by the two-dimensional mood scale. Although no significant difference between the groups was observed for any attribute, the Glycerol group tended to show lower values in positive arousal during hydration than the Placebo group. The difference faded away $60 \mathrm{~min}$ after the end of hydration, while 

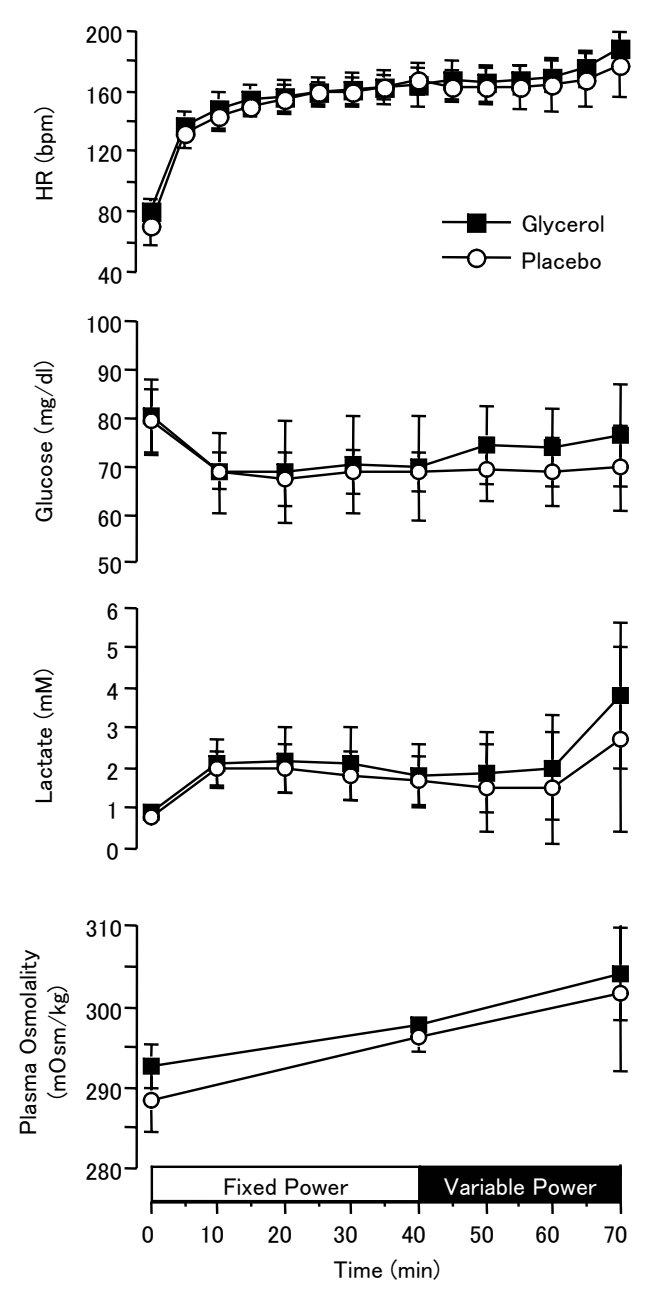

Figure 5 Changes in heart rate (HR), glucose, lactate and plasma osmolality in glycerol and placebo performance trials. Data are expressed as mean $\pm \operatorname{SD}(n=6)$.

the Glycerol group in turn showed higher values in positive arousal during subsequent exercise. The values for negative arousal and arousal increased during exercise and decreased in hedonic. In addition, the psychological state of the subjects on the experiment day as recorded through POMS did not show any significant difference between groups for any items (tension, depression, anger, energy, fatigue, confusion and comprehensive unpleasant index). Almost all subjects reported drowsiness during the glycerol fluid intake but no one reported any indefinite complaint (such as a minor headache).

\section{Discussion}

\subsection{Examining water retention effect due to different glycerol loading methods}

It has been already suggested that the water
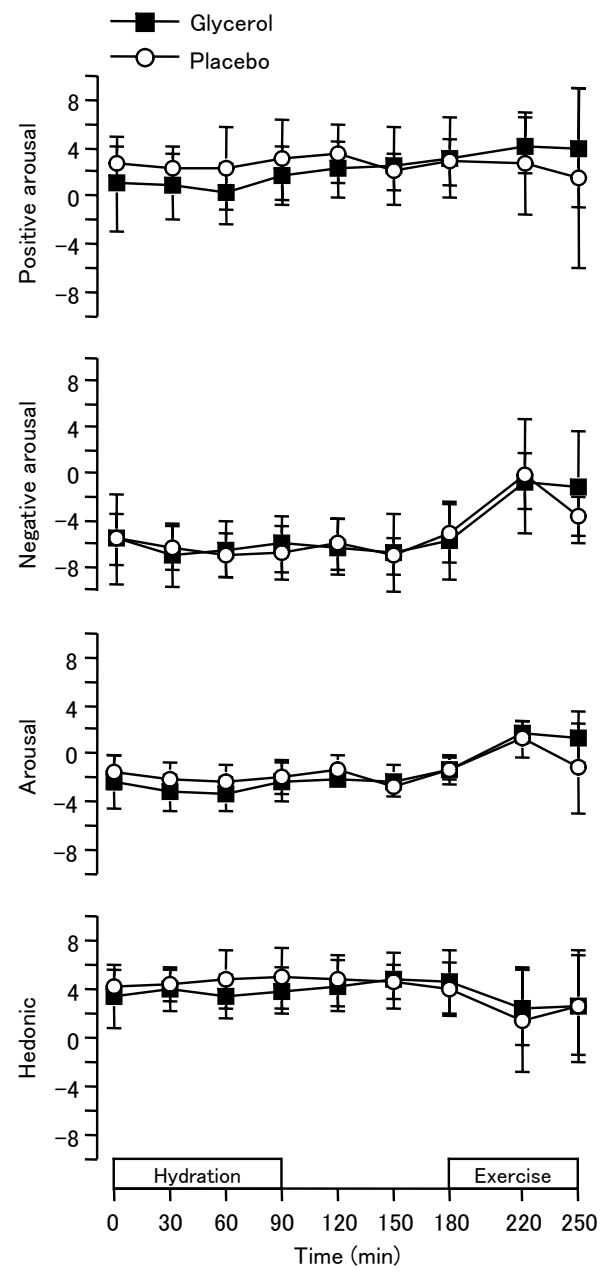

Figure 6 Changes in positive arousal, negative arousal, arousal, and hedonic measured by two-dimensional mood scale in glycerol and placebo trials over time. Data are expressed as mean $\pm \operatorname{SD}(n=6)$.

retention effect of glycerol loading varies due to differences in water intake methods (Robergs and Griffin, 1998) but no study has compared these differences within the same experiment. Thus, the water retention effects of two different glycerol loading methods were investigated in the same subject in Experiment 1. The methods used in the experiment were: the Bolus-G protocol as used by Montner et al. (1996) and which is regarded as most effective in water retention; the $\mathrm{G}$ protocol whose effect is estimated to be second to Bolus-G; and, the HW protocol was used as a control which did not contain glycerol.

Significant water retention effects of glycerol loading were observed in this study. Nude body weight significantly increased and urine volume significantly decreased, both in the $\mathrm{G}$ and Bolus-G protocols compared to the HW protocol. However, no significant difference in changes in body weight 
and urine volume were observed between $G$ and Bolus-G protocols. It is suggested that the difference in these hydration protocols might not have any effect on water retention. However, the water retention effect of the Bolus-G protocol was more than double that of the effect of the $G$ protocol in four out of ten subjects, indicating that water retention effects due to differences in hydration methods do vary according to individual variability and physical condition at hydration.

The increase in body water volume due to glycerol loading is ascribed to a decrease in urine volume, the mechanism of which has been investigated in detail by Freund et al. (1995). Firstly, the involvement of antidiuretic hormone (ADH) was examined. Although glycerol loading significantly increased plasma osmolality, it significantly decreased plasma $\mathrm{ADH}$ concentration in a similar way as when a drink without glycerol was taken. Since glycerol acts weakly against osmoreceptors in supraoptic nucleus, it seems that an increase in plasma osmolality due to glycerol fluid intake would not raise ADH secretion. In contrast, the water retention effect of glycerol loading is considered to be caused not by the ADH-mediated function but by the immediate effect of glycerol on the osmotic pressure gradient of the renal medulla. This promotes water reabsorption because glycerol is actively reabsorbed by the medulla of kidney (Freund et al., 1995).

\subsection{Effect of glycerol loading on dehydration and exercise performance during exercise under hot conditions}

Whereas glycerol loading yields hyperhydration, an agreement has not been achieved on whether glycerol loading alleviates dehydration and improves exercise performance during exercise under hot conditions. Thus, the amount of dehydration and exercise performance were examined during 70-min cycling exercise under hot conditions in Experiment 2. The Bolus-G protocol, which had a comparatively higher water retention effect in Experiment 1, was adopted as the method of glycerol loading.

Body weight before exercise increased by 920 $\pm 229 \mathrm{~g}$ in the Glycerol group with the difference being $790 \mathrm{~g}$ compared to the Placebo group. When comparing the water retention amount with previous studies (around 300-700g), it can be stated that high level hyperhydration before exercise was achieved. Heavy sweating was observed during the 70-min cycling exercise and body weight decreased significantly in both the Glycerol and Placebo groups. The influence of water loss caused by urination can not be excluded because urine volume immediately after exercise was not measured in this study, but no significant difference in shrinkage of body weight caused by exercise was observed between the groups, and it is presumed that there was little difference in sweating volume during exercise. The dehydration volume (relative volume per body weight) compared to pre-hydration was $1,680 \mathrm{~g}$ in the Placebo group (2.5\% of body weight), compared to $890 \mathrm{~g}(1.4 \%$ of body weight) in the Glycerol group $(p<0.01)$, which shows that glycerol loading could alleviate dehydration during exercise. Walsh et al. (1994) report that a decline of body water volume of only $1.8 \%$ of body weight can significantly shorten the ability of athletes to undergo high intensity cycling. Hydration is recommended during exercise under hot conditions so that body weight shrinkage does not exceed $2 \%$ of body weight (Kawahara and Morimoto, 1994) (Guidebook to prevent heat prostration during exercise published by the Japan Sports Association (JASA)). Although subjects were not hydrated during exercise in this study, it is expected that hydration during exercise combined with glycerol loading might lead to an increased alleviation of dehydration.

Potential performance improvements during exercise due to glycerol loading were also examined in this study. The cycling exercise test consisted of a 40-min fixed power phase and a 30-min variable power phase with performance being evaluated by the total work load at the variable power phase. Hitchins et al. (1999) report improved exercise performance due to glycerol loading using a similar protocol. In this experiment, no significant difference was observed, although the total work load increased by an average $9 \%$ in the Glycerol group. It is presumed that the cause of relatively low performance improvements is because of large individual differences and the fact that the subjects were inexperienced in cycling as they were middle-distance athletes. Several previous studies report an improvement in exercise performance due to glycerol loading (Montner et al., 1996 ; Hitchins et al., 1999 ; Anderson et al., 2001). Although this study did not show any improvement effect due to 
glycerol loading, it might be observed depending on the exercise conditions (temperature, humidity, exercise genre and movement time). This is to be investigated in future, as well as conducting a similar study with an increased number of subjects.

When body water volume decreases during exercise, colon and muscle temperature increases (Hargreaves et al., 1996), heart rate increases (Armstrong et al., 1985), cardiac output declines (Hamilton et al., 1991) and cutaneous blood flow declines (Gonzalez-Alonso et al., 1999), which leads to a deterioration in exercise performance. Moreover, severe dehydration raises the danger of heat stroke. This is a very important issue in Japan which has a hot and humid summer which has caused heat stroke fatalities during exercise. The effect of glycerol loading on alleviating dehydration during exercise was acknowledged in this study, although glycerol loading did not specifically improve exercise performance. Therefore, it is assumed that glycerol loading might be one potential measure for effective conditioning in order to prevent dehydration and heat stroke during exercise under hot conditions.

\subsection{Effect of glycerol loading on mood}

There were several subjects who reported minor headaches and blurred vision during glycerol fluid intake in Experiment 1.which indicates the possible negative effects of glycerol loading on pre-exercise mood. Therefore, changes in mood during hydration and during exercise were examined Experiment 2 using a two-dimensional mood scale.

Although significant differences were not observed in positive arousal, the Glycerol group showed lower values than the Placebo group. The fact that most subjects reported drowsiness during glycerol fluid intake in Experiment 2 might suggest that glycerol fluid intake could decrease positive arousal by causing drowsiness. However, given that no significant difference in mood immediately before exercise was observed between the groups, and that the symptoms of all the subjects who reported complaints in Experiment 1 resolved 60 min after the fluid intake was completed, it is presumed that any effect on pre-exercise mood can be ignored by setting a rest period of about $60 \mathrm{~min}$. It would seem sensible to confirm beforehand whether glycerol suits an individual's constitution by trial intake before conducting glycerol loading.
Fatigue and heavy sweating during exercise increased the occurrence of negative moods such as "irritating" and "straining". Levels of negative arousal and arousal and a decrease in levels of hedonic were also observed. No statistically significant difference was observed $40 \mathrm{~min}$ after starting exercise and the difference in values was small; higher values were observed regarding positive arousal in the Glycerol group. Gopinathan et al. (1988) claim that dehydration affects psychological states, and we hypothesize that glycerol loading might possibly maintain positive arousal during exercise by alleviating dehydration during exercise.

In summary, this study investigated water retention effects according to different glycerol loading methods (Experiment 1), and the impact of glycerol loading on the alleviation of dehydration during longtime exercise under hot conditions, combined with its effect on mood and exercise performance (Experiment 2). We demonstrated that difference in protocols of the two glycerol loading methods used in this study might not affect water retention effect. However, since the water retention effect showed individual differences, it seems necessary to confirm an effective loading method for each individual before adopting it in a competitive game. Glycerol loading effectively alleviated dehydration caused by exercise under hot conditions in this study without affecting mood during hydration and during exercise. Although this study could not reach a definite conclusion on whether glycerol loading would improve exercise performance under hot conditions because of individual differences, further investigation is necessary in other conditions, for instance, extending exercise time and increasing dehydration levels.

\section{References}

Anderson, M.J., Cotter, J.D., Garnham, A.P., Casley, D.J. \& Febbraio, M.A. (2001) Effect of glycerol-induced hyperhydration on thermoregulation and metabolism during exercise in heat. Int. J. Sport Nutr. Exerc. Metab., 11: 315-333.

Armstrong, L.E., Costill, D.L. \& Fink, W.J. (1985) Influence of diuretic-induced dehydration on competitive running performance. Med. Sci. Sports Exerc., 17: 456-461.

Beaver, W.L., Wasserman, K. \& Whipp, B.J. (1985) Improved detection of lactate threshold during exercise using a log-log transformation. J. Appl. Physiol., 59: 1936-1940.

Borg, G. (1970) Perceived exertion as an indicator of somatic stress. Scand. J. Rehabil. Med., 2: 92-98.

Burke, L.M. \& Hawley, J.A. (1997) Fluid balance in team sports. 
Guidelines for optimal practices. Sports Med., 24: 38-54.

Burke, L.M. (2001) Nutritional needs for exercise in the heat. Comp. Biochem. Physiol., 128: 735-748.

Freund, B.J., Montain, S.J., Young, A.J., Sawka, M.N., DeLuca, J.P., Pandolf, K.B. \& Valeri. C.R. (1995) Glycerol hyperhydration: hormonal, renal, and vascular fluid responses. J. Appl. Physiol., 79: 2069-2077.

Gonzalez-Alonso, J., Mora-Rodriguez, R., Below, P.R. and Coyle, E.F. (1995) Dehydration reduces cardiac output and increases systemic and cutaneous vascular resistance during exercise. J. Appl. Physiol., 79: 1487-1496.

Gopinathan, P.M., Pichan, G. \& Sharma, V.M. (1988) Role of dehydration in heat stress-induced variations in mental performance. Arch. Environ. Health, 43: 15-17.

Hamilton, M.T., Gonzalez-Alonso, J., Montain, S.J. \& Coyle, E.F. Fluid replacement and glucose infusion during exercise prevent cardiovascular drift. J. Appl. Physiol., 71: 871-877.

Hargreaves, M., Dillo, P., Angus, D. \& Febbraio, M. (1996) Effect of fluid ingestion on muscle metabolism during prolonged exercise. J. Appl. Physiol., 80: 363-366.

Hitchins, S., Martin, D.T., Burke, L., Yates, K., Fallon, K., Hahn, A. \& Dobson, G.P. (1999) Glycerol hyperhydration improves cycle time trial performance in hot humid conditions. Eur. J. Appl. Physiol. Occup. Physiol., 80: 494-501.

Jeukendrup, A., Saris, W.H., Brouns, F. \& Kester A.D. (1996) A new validated endurance performance test. Med. Sci. Sports Exerc., 28: 266-270.

Kawahara, T. \& Morimoto, T. (1999) Guidebook for preventing heat disorder during sports. Tokyo: Japan Sports Association.(in Japanese)

Lyons, T.P., Riedesel, M.L., Meuli, L.E. \& Chick, T.W. (1990) Effects of glycerol-induced hyperhydration prior to exercise in the heat on sweating and core temperature. Med. Sci. Sports. Exerc., 22: 477-483.

Marino, F.E., Kay, D. \& Cannon, J. (2003) Glycerol hyperhydration fails to improve endurance performance and thermoregulation in humans in a warm humid environment. Pflugers Arch., 446: 455-462.

McConell, G.K., Burge, C.M., Skinner, S.L. \& Hargreaves, M. (1997) Influence of ingested fluid volume on physiological responses during prolonged exercise. Acta. Physiol. Scand., 160: 149-156.

Montner, P., Stark, D.M., Riedesel, M.L., Murata, G., Robergs, R., Timms, M. \& Chick, T.W. (1996) Pre-exercise glycerol hydration improves cycling endurance time. Int. J. Sports Med., 17: 27-33.

Murray, R., Eddy, D.E., Paul, G.L., Seifert, J.G. \& Halaby, G.A. (1991) Physiological responses to glycerol ingestion during exercise. J. Appl. Physiol., 71: 144-149.

Riedesel, M.L., Allen, D.Y., Peake, G.T. \& Al-Qattan, K. (1987) Hyperhydration with glycerol solutions. J. Appl. Physiol., 63: 2262-2268.

Robergs, R.A. \& Griffin, S.E. (1998) Glycerol. Biochemistry, pharmacokinetics and clinical and practical applications. Sports Med., 26: 145-167.

Sakairi, Y., Tokuda, H., Kawahara, M., Yagi, T. \& Soya, H. (2003a) Development of the two dimension mood scale for measuring psychological arousal level and hedonic tone. Bull. Inst. Health \& Sport Sci., Univ. of Tsukuba, 26: 27-36. (in Japanese)

Sakairi, Y. \& Soya, H. (2003b) Novel psychological scale for monitoring mood during exercise. Taiikunokagaku, 53:
845-850. (in Japanese)

Sawka, M.N. (1992) Physiological consequences of hypohydration: exercise performance and thermoregulation. Med. Sci. Sports Exerc., 24: 657-670.

Sawka, M.N., Montain, S.J. \& Latzka, W.A. (2001) Hydration effects on thermoregulation and performance in the heat. Comp. Biochem. Physiol., 128: 679-690.

Walsh, R.M., Noakes, T.D., Hawley, J.A. \& Dennis, S.C. (1994) Impaired high-intensity cycling performance time at low levels of dehydration. Int. J. Sports Med., 15: 392-398.

Yorimoto, A., Nakai, S., Yoshida, T. \& Morimoto, T. (1995) Relationship between drinking behavior and body temperature during exercise in heat. Jpn. J. Phys. Fitness Sports Med, 44: 357-364.(in Japanese)

Young, A.J., Sawka, M.N., Epstein, Y., Decristofano, B. \& Pandolf, K.B. (1987) Cooling different body surfaces during upper and lower body exercise. J. Appl. Physiol., 63: 1218-1223.

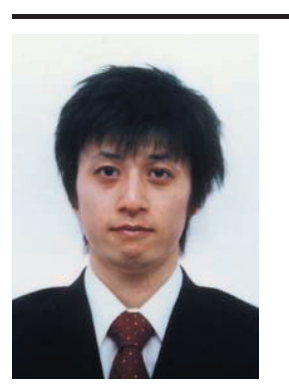

Name:

Takeshi Nishijima

Affiliation:

Exercise Biochemistry, Graduate School of Comprehensive Human Sciences, University of Tsukuba

Address:

1-1-1 Tennodai, Tsukuba, Ibaraki 305-8574 Japan

Brief Biographical History:

2001-2006 Ph.D. (Health and Sport Sciences), Doctoral Program of Health and Sport Sciences, Graduate School of Comprehensive Human Sciences, University of Tsukuba.

2006- Present Researcher (COE), Graduate School of Comprehensive Human Sciences, University of Tsukuba.

Main Works:

- Nishijima T, Soya H, Evidence of functional hyperemia in the rat hippocampus during mild treadmill running. Neurosci Res, 54: 186-191, 2006.

- Nishijima T, Soya H, Hemodynamics under hippocampal functional hyperemia in anesthesized rats: a greater contribution of red blood cells velocity compared to its concentration. Jpn J Physiol, 55: 303-307, 2005.

Membership in Learned Societies:

- Society for Neuroscience

- The Japan Neuroscience Society

- Japanese Society of Physical Fitness and Sports Medicine

- Japan Society of Physical Education, Health and Sport Sciences 